\title{
Automated Human Identification Using Ear Imaging
}

\author{
Pankti Mehta ${ }^{1}$, Vrudhhi Shah ${ }^{2}$, Mansingh Rathod $^{3}$ \\ Department of Information Technology, K.J.Somaiya Institute of Engineering And Information Technology, Sion, Mumbai, India
}

Professor, Department of Information Technology, K.J.Somaiya Institute of Engineering and Information Technology, Sion, Mumbai, India

\begin{abstract}
Ear is a new area of advance biometrics that remains unchanged from childhood to early old age. It is unaffected by facial expressions, cosmetics and eye glasses. Nowadays, biometric system plays a important role in almost all the security aspects. As it uses earmark for the identification purpose which is impossible to steal or lose, they provide a better solution to pins and passwords. Automated identification through biometrics especially palm and iris recognition have been extensively used. An alternative to this is ear biometrics. It has been seen that finding two ears which are completely identical is almost impossible and ear does not change much with time, Moreover, ear satisfies all the properties that should be possessed by a biometric.[4]. The outer ear has been established as a stable and unique biometric characteristic. In previous years, increasing efforts have been made for creating an automated authentication system utilizing the outer ear. One important processing step in these systems is the detection of the ear region. Automated ear detection faces a number of challenges, such as invariant processing of both left and right ears, as well as the handling of occlusion and pose variations. We propose a new approach for the detection of ears, which uses features from texture and depth images, as well as context information. With a detection rate of $90 \%$ on profile images, our approach is highly reliable. Moreover, it is invariant to rotations and it can detect left and right ears. We also show, that our method is working under realistic conditions by providing simulation results on a more challenging dataset, which contains images of occluded ears from various poses.
\end{abstract}

Keywords: Biometrics, morphological operators, watershed algorithm, Gabor filters

\section{Introduction}

IN recent years, biometrics has been receiving a lot of attention. Human ear is a perfect data for passive person identification, which can be applied to provide security in the public places. The ear has desirable properties such as universality, uniqueness and permanence [6]. There are many human traits that can be used as a biometric like fingerprint, face, voice and iris. Despite extensive research many problems in these remain largely unsolved a wide variety of imaging problems (e.g., lighting, shadows, scale, and translation) [4].

The outer ear is believed to be just as unique as the face. The capability of automatically establishing the identity of individuals, called as verification or identification is reliable for several applications such as access control and data systems.

\section{Existing System}

The utility of Iannarelli's approach for the automated ear recognition is quite limited since this approach required accurate estimation of a stable reference points for measurements, which is very difficult in real environment. the work is largely conceptual and lacks experimental results on any ear database.

This paper presents a completely automated approach for the personal identification using 2D ear imaging. Automated and accurate segmentation of exact region of interest, from the acquired gray level ear images, is quite difficult.

However the success of the feature extractor and the achievable accuracy/ performance significantly depends on Biometrics refers to metrics related to human characteristics.
Biometrics authentication (or realistic authentication) is used in computer science as a form of identification and access control. It is also used to identify individuals in groups that are under surveillance.

It has magnitudal advantages over traditional authentication techniques namely passwords, ID card and key, due to biometric characteristics of an individual are not transferable and unique for every person. Like any other biometric identifiers, ears are believed to have the desirable properties of universality, uniqueness, permanence and collectability for personal recognition. In addition, there are several motivations for ear biometric Firstly, the ear data can be captured using conventional cameras. Secondly, the data collection is nonintrusive (i.e., requires no cooperation from the user). Thirdly, ear based access systems are very suitable for several usages. Finally, ear features are more stable over time and are not susceptible to major changes. The accuracy for such automated ear segmentation.

Therefore significant efforts are required to develop robust techniques for the completely automated ear segmentation. We present a new approach for the automated ear segmentation which has been proven to be quite effective in achieving robust segmentation of the curved region of interest. Our ear segmentation approach effectively utilizes combinations of morphological operations and Fourier descriptors to achieve reliable ear segmentation.[2]

\section{Proposed System}

- The overall design of this system consists of six major modules describes the flow between these modules.[1]

- Once the ear soft image is converted in to a jpeg, the image will be uploaded to the system. 


\section{International Journal of Science and Research (IJSR) \\ ISSN (Online): 2319-7064}

Index Copernicus Value (2013): 6.14 | Impact Factor (2014): 5.611

- Then in the next module, image will be preprocessed in order to get a clear image to be processed in the segmentation module.

- The image segmentation module will isolate the objects in the brain image, in order to extract features of each object in the next module.

- Set of image processing activities will be carried out to super impose the image with the required features.

- Then the unnecessary noise and objects will be removed if it is needed and will mark the objects that will be used to extract features.

- The features will be extracted to feed the neural network as an input to train or recognize the type of the hemorrhage.

- Finally the type of the hemorrhage will be identified according to the trained neural network which is being created in the training phase of the system. Once the network is created, saved network can once the input features are extracted and output result is created network

- If the user is satisfied with the result, user will system to gain better output the next time. A new successful training is done to decide whether the training percentage is better or to the system with the same input and output features

Following the flowchart for this project following steps steps are completed and the result after each step is shows in experimental results.

\section{Pre-processing}

Image is preprocessed by performing following steps:

1) Resizing the image is done so it fits on the system user interface.

2) Convert in to gray scale image to make it contrast

3) Convert in to two dimensional images.

4) To gain better output the next time. A new network file will be created once a training is done.

According to the results generated in the plot, user will be able whether the training percentage is better or to try out once again just by training with the same input and output features.

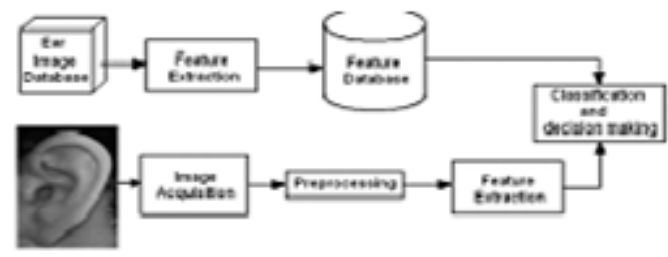

Segmentation Using Morphological Operations:

1) MorphologicalTechniques

a) Opening- by-reconstruction

b) Closing- by-reconstruction

c) Complement image

d) Calculate regional maxima

e) Superimpose the image

2) Compute Background Markers

3) Watershed Transformation and the Once the network is created, saved network can be used for training of the next images input features are extracted and output result is generated using the previously created network file. If the user is satisfied with the result, user will be able to add the test image to train Segmentation

4) Visualize the Result

5) Remove Background Noise

The image will be segmented to identify the relevant objects in the CT scan and to retrieve the values needed as input. Once the image is segmented properly, required information will be extracted to feed the neural network.

\section{Morphological Operations}

The basic mathematical morphological operators are dilation and erosion and other morphological operations are synthesized from these two basic operations.

Let $\mathrm{F}(\mathrm{x}, \mathrm{y})$ denote a gray-scale two dimensional image and A denote structuring element. Dilation of a gray-scale image $\mathrm{F}(\mathrm{x}, \mathrm{y})$ by a grayscale structuring element $\mathrm{A}(\mathrm{s}, \mathrm{t})$ is denoted by:

$(\mathrm{F} \oplus \mathrm{A})(\mathrm{x}, \mathrm{y})=\max \llbracket \mathrm{F}(\mathrm{x}-\mathrm{s}, \mathrm{y}-\mathrm{t})+\mathrm{A}(\mathrm{s}, \mathrm{t}) \rrbracket$

Erosion of a gray-scale image $\mathrm{F}(\mathrm{x}, \mathrm{y})$ by a gray-scale structuring element $A(s, t)$ is Opening and closing of grayscale image $F(x, y)$ by gray-scale structuring element $A(s, t)$ are denoted respectively bydenoted by Opening and closing of gray-scale image $\mathrm{F}(\mathrm{x}, \mathrm{y})$ by gray-scale structuring element $\mathrm{A}(\mathrm{s}, \mathrm{t})$ are denoted respectively by:

$(\mathrm{F} \ominus \mathrm{A})(\mathrm{x}, \mathrm{y})=\min \mathrm{F}(\mathrm{x}-\mathrm{s}, \mathrm{y}-\mathrm{t})-\mathrm{A}(\mathrm{s}, \mathrm{t})$

$\mathrm{F} \odot \mathrm{A}=(\mathrm{F} \ominus \mathrm{A}) \oplus \mathrm{A}$

$\mathrm{F} \odot \mathrm{A}=(\mathrm{F} \oplus \mathrm{A}) \ominus \mathrm{A}$

\section{Edge Based Segmentation Method}

Edge-base segmentation generally indicates the segmentation method based on the edge in an image. The simple methods apply some edge detection methods before segmentation. Some edge detection methods are gradient operators and Hilbert transform.

Then the other methods only base on the concept of edge instead of using edge detection methods, for instance, watershed segmentation algorithm.

\section{Markers}

For resolving the over-segmentation problem in the watershed algorithm, an approach based on the concept of marker is described in [1]. A marker is a connected component belonging to an image. The markers include the internal markers, associated with objects of interest, and the external markers, associated with the back-ground. The marker selection typically consists of two steps: preprocessing and definition of a set of criteria that markers must satisfy. The pre-processing scheme is to filter an image with a smoothing filter. This step can minimize the effect of small spatial detail, in other words, this step is to reduce the large number of potential minima (irrelevant detail), which is the reason of over segmentation. The definitions of an internal marker is :

a) A region that is surrounded by points of higher "altitude".

b) The points in the region form a connected component.

c) All the points in the connected component have the same intensity value. 


\section{International Journal of Science and Research (IJSR) \\ ISSN (Online): 2319-7064}

Index Copernicus Value (2013): 6.14 | Impact Factor (2014): 5.611

After the image is smoothed, the internal markers can be defined by these definitions, shown as light gray, blob like regions. Consequently, the watershed algorithm is applied to the smoothed image, under the restriction that these internal markers be the only allowed regional minima. The watershed lines, defined as the external markers.

The points of the watershed line are along the highest points between neighboring markers. The external markers effectively segment the image into several regions with each region composed by a single internal marker and part of the background. Then the goal is to reduce each of these regions into two: a single object and its background. The segmentation techniques discussed earlier can be applied to each individual region. The segmentation result of applying the watershed algorithm to each image.

\section{Conclusion}

Detecting the image is very crucial step in the security system of the Organisation. Automatic detection of image is a very complex task. The segmentation and the quantification of region is based on the watershed algorithm based segmentation procedure. After making the use of watershed algorithm its advantages and disadvantages are as follows:

\section{Advantages}

1) The boundaries of each region are continuous.

\section{Disadvantages}

1) The segmentation result has over-segmentation problem

2) The algorithm is time-consuming.

For resolving the over-segmentation problem in the watershed algorithm, an approach based on the concept of marker is used. A marker is a connected component belonging to an image. Before application of the watershed algorithm morphological operations are performed to compute the foreground and background markers.

\section{References}

[1] Harshawardhan P. Ahire, Jagruti K. Save Brain Hemorrhage Detection using Artificial Neural Network International Journal of Informatics and Communication Technology (IJ-ICT) 2013

[2] Automated human identification using ear imagingAjay Kumar nDepartment of Computing, The Hong Kong Polytechnic University, Hung Hom, Kowloon, Hong Kong, Chenye Wu 2011

[3] Robust Localization of Ears by Feature Level Fusion and Context InformationAnikaPflug, Adrian Winterstein, ChristophBuschda/sec - Biometrics and Internet Security Research GroupHochschule Darmstadt, Germany

[4] Proceedings of icetect 2011 978-1-4244-7926$9 / 11 / \$ 26.00 \quad$ C)2011 ieeear based attendance monitoringsystemmr.jitendra b. jawale,dr. smt.anjali s. bhalchandra, proceedings 0 f icetect 2011 